\title{
Etileno y retardantes de la maduración en la poscosecha de productos agrícolas. Una revisión
}

\section{Ethylene and maturation retardants in the postharvest of perishable horticultural products. A review}

HELBER ENRIQUE BALAGUERA-LÓPEZ1, 5

FREDY ALEXÁNDER SALAMANCA-

GUTIÉRREZ (q.e.p.d.) $)^{2}$

JUAN CAMILO GARCÍA ${ }^{3}$

ANÍBAL HERRERA-ARÉVALO ${ }^{4}$

Frutos de banano maduro

(izquierda) y verde (derecha) a

los 21 días de almacenamiento,

tratados con 1-metilciclopropeno.

Foto: J.C. García

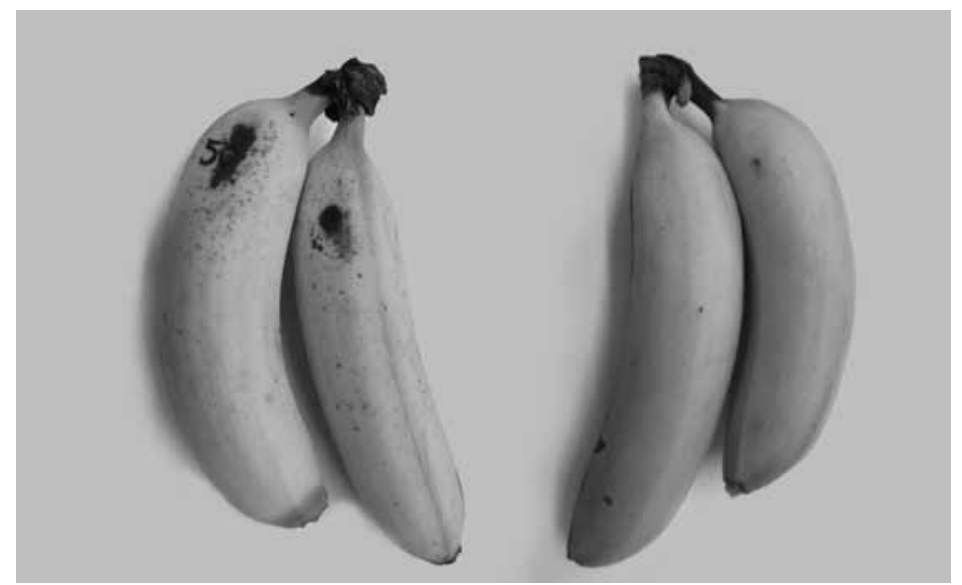

\section{RESUMEN}

El etileno es la hormona vegetal responsable de regular diferentes procesos durante la maduración de productos agrícolas, llevando a procesos de senescencia y finalmente, pérdida de valor nutricional y comercial. A través del tiempo se han desarrollado diversos retardantes químicos de la maduración para disminuir los efectos negativos del etileno en la poscosecha, los cuales actúan en las etapas de biosíntesis (aminoetoxi-vinil-glicina (AVG) y ácido aminooxiacético (AOA), acción/señalización (1-MCP)) y sales de plata como nitrato y tiosulfato de plata (STS) y compuestos que oxidan al etileno de la atmósfera (permanganato de potasio $\left(\mathrm{KMnO}_{4}\right)$ ). En esta revisión se describen las características principales de cada uno de los retardantes de madurez mencionados, además, se analiza con estudios científicos, el efecto que tienen estos retardantes sobre los cambios moleculares, fisiológicos, químicos y físicos durante la poscosecha de frutos, hortalizas y especies ornamentales.

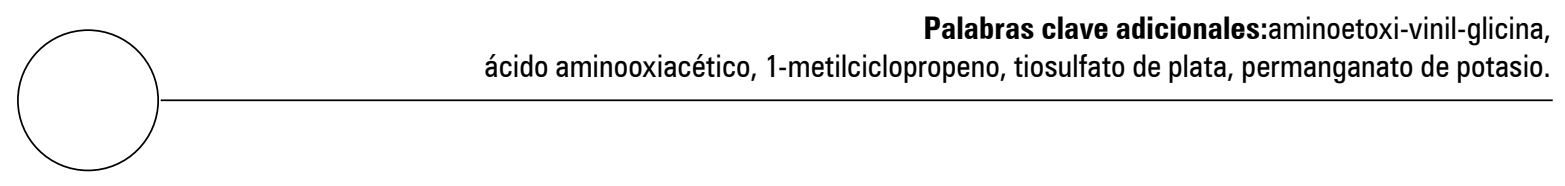

1 Facultad de Ciencias Agrarias, Programa de Doctorado en Ciencias Agrarias, Línea Fisiología de Cultivos, Universidad Nacional de Colombia, Bogotá (Colombia).

2 Facultad de Ciencias Agrarias, Universidad Nacional de Colombia, Bogotá (Colombia).

3 Facultad de Ciencias Agrarias, Programa de Maestría en Ciencias Agrarias, Línea Fisiología de Cultivos, Universidad Nacional de Colombia, Bogotá (Colombia).

4 Facultad de Ciencias Agrarias, Departamento de Agronomía, Universidad Nacional de Colombia, Bogotá (Colombia).

5 Autor para correspondencia. hebalagueral@unal.edu.co 


\section{ABSTRACT}

Ethylene is the plant hormone responsible for regulating various processes during the ripening of agricultural products, leading to a senescence process and, finally, to a loss of the nutritional and commercial values. Over time, different chemical maturation retardants have been developed to decrease the negative effects of ethylene, which act on the stages of biosynthesis (aminoethoxy-vinyl-glycine (AVG) and aminooxyacetic acid (AOA), action/signaling (1-methylcyclopropene (1-MCP)) and silver salts, such as nitrate and silver thiosulfate (STS) and compounds that oxidize the ethylene of the atmosphere (potassium permanganate $\left(\mathrm{KMnO}_{4}\right)$. In this review, the main characteristics of each chemical retardant are discussed along with the scientific studies on the effects of these retardants on the molecular, physiological, chemical and physical changes during the postharvest of fruits, vegetables and ornamental species.

Additional key words: aminoethoxy-vinyl glycine, aminooxyacetic acid, 1-methylcyclopropene, silver thiosulfate, potassium permanganate.

Fecha de recepción: 10-07-2014

Aprobado para publicación: 10-11-2014
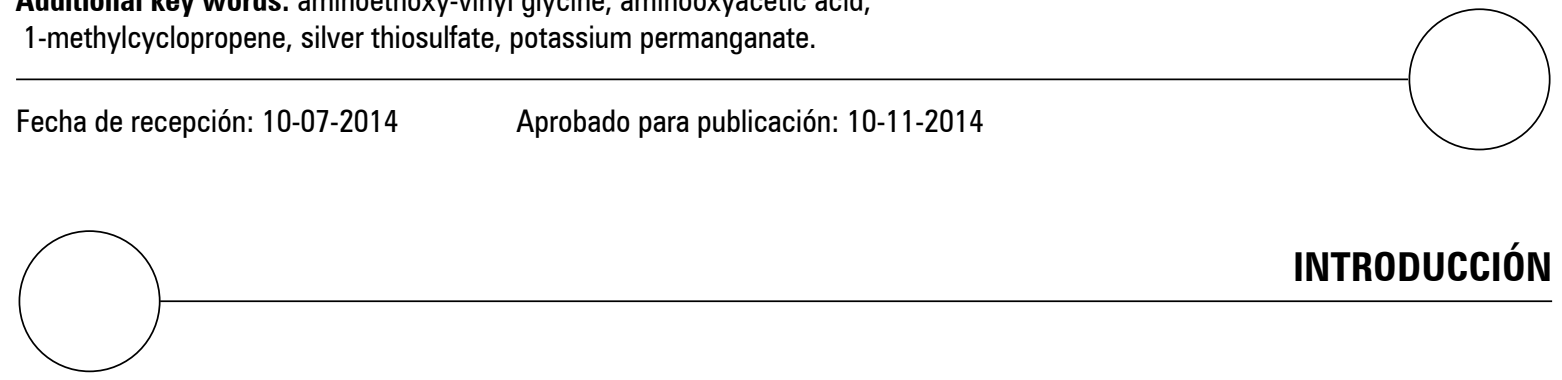

El etileno regula la maduración y senescencia de productos agrícolas a nivel molecular, bioquímico y fisiológico (Kesari et al., 2007), debido a que estimula la expresión de genes que codifican para las enzimas relacionadas con los cambios durante la maduración y/o senescencia (Jiang y Fu, 2000). El etileno tiene un papel doble en la poscosecha, por un lado ocasiona que los frutos adquieran características organolépticas óptimas para su consumo, pero también es responsable de la senescencia de los tejidos, generando efectos desfavorables en la calidad (Bapat et al., 2010).

El conocimiento del mecanismo de acción del etileno ha permitido generar diferentes tecnologías y procedimientos para disminuir sus efectos negativos, donde se incluye la refrigeración, uso de atmósferas modificadas y controladas, aplicación de calcio, entre otros; sin embargo, los retardantes químicos de madurez han mostrado mayor eficiencia en el control de la madurez y senescencia de frutos, hortalizas y flores (Arora et al., 2008).

Los retardantes químicos de la maduración se pueden clasificar en inhibidores de la sín- tesis del etileno, donde se incluyen productos como aminoetoxi-vinil-glicina (AVG) y ácido aminooxiacético (AOA); inhibidores de la señalización (acción) del etileno, como 1-metilciclopropeno (1-MCP) y sales de plata (nitrato y tiosulfato de plata) (Serek et al., 2006), entre otros. Otro grupo son los oxidantes de etileno, siendo el permanganato de potasio $\mathrm{KMnO}_{4}$ el producto más importante (Wills y Warton, 2004). El objetivo de esta revisión es realizar el estado del arte del etileno y el uso de estos retardantes de madurez en productos hortícolas perecederos como frutos, hortalizas y flores, destacando algunas generalidades, modo de acción, utilidades, desventajas, dosis, productos comerciales y algunas restricciones que pueden presentar.

\section{Biosíntesis de etileno}

El etileno se sintetiza a partir del aminoacido metionina, primero se da la conversión de metionina a S-adenosil-L-metionina (SAM) catalizada por la enzima SAM sintetasa, luego se presenta la formación de 1-aminociclopropano-1-ácido carboxílico (ACC) a partir de 
SAM mediante la enzima ACC sintasa (ACS) y finalmente la conversión de ACC a etileno, catalizada por la ACC oxidasa (ACO) (Bapat et al., 2010). La metionina es reciclada en el ciclo de "Yang" lo que conlleva a tener altas tasas de producción de etileno sin necesidad de altos niveles de metionina intracelular (Barry y Giovannoni, 2007).

\section{Señalización}

La señalización inicia con la unión del etileno a un grupo de receptores proteicos presentes en la membrana del retículo endoplasmático (Chen et al., 2005), los cuales son codificados por una familia multigénica que produce proteínas estructuralmente diferentes pero con una función redundante (Chen et al., 2005; Bouzayen et al., 2010). La unión etileno-receptor ocurre en el dominio $\mathrm{N}$-terminal del receptor y requiere iones de $\mathrm{Cu}$ (I) para formar un dímero de receptor (Binder, 2008). Los genes que codifican para los receptores se expresan diferencialmente dependiendo del órgano, tejido, etapa de desarrollo y en respuesta a estímulos exógenos (Bouzayen et al., 2010). El número de receptores varía entre especies, por ejemplo, en Arabidopsis se han reportado 5 receptores (ETR1 y 2 (ETHYLENERESPONSE); ERS1 y 2 (ETHYLENE-SENSOR) y EIN4 (ETHYLENE-INSENSITIVE) (Klee y Giovannoni, 2011); en tomate 7 (ETR1 al 7, donde ETR3 se conoce como NR (NEVER-RIPE)) (Barry y Giovannoni, 2007) y en manzana 5 (ETR1-2-5 y ERS1-2) (Yang et al., 2013).

El siguiente componente en la vía de señalización es CTR1 (CONSTITUTIVE-TRIPLERESPONSE1), la cual es una proteína quinasa Raf-like ser/thr con similitud en su dominio C-terminal a la proteína MAPKKK (mitogen-activated-protein kinase-kinase-kinase) encontrada en mamíferos (Chen et al., 2005; Czarny et al., 2006). Ésta proteína interactúa con el dominio quinasa de los receptores y en presencia de etileno desencadena una cascada de fosforilaciones MAP-quinasa (Adams-Phillips et al., 2004) que afectan el estado de fosforilación de otras proteínas corriente abajo, incluyendo factores de transcripción (Stepanova y Alonso, 2009).

Corriente abajo de CTR1, se encuentra EIN2 (ETHYLENE INSENSITIVE2), la cual es una proteína de membrana transportadora de metales, con similitud a transportadores tipo NRAMP (NATURAL-RESISTANCE ASSOCIATED-MACROPHAGE-PROTEIN) (Czarny et al., 2006). La función de EIN2 es regular la disponibilidad del factor de transcripción EIN3 mediante un mecanismo hasta el momento desconocido (Sang-Dong et al., 2009). En respuesta al etileno, EIN3 y EIL1 (EIN3-LIKE1) se unen a un motivo (motif) conocido como elemento primario de respuesta al etileno (PERE), que está presente en los promotores de ERF1 (ETHYLENE-RESPONSE-FACTOR1), EBF2 (EIN3-BINDING-SITES) y varios genes relacionados con la senescencia y maduración de frutos (Barry y Giovannoni, 2007). Lo anterior lleva al incremento de la expresión de genes relacionados con la maduración, como, ACS2, ACS4 en tomate (Klee y Giovannoni, 2011); ACS2 en brócoli (Gang et al., 2009); ACO y EIN2 en manzana (Yang et al., 2013); ACO en durazno (Zhang et al., 2012) XET y EXP (xiloglucano-endotransglicosilasa y expansinas) en chirimoya (Li et al., 2009), entre otros.

\section{El 1-Metilciclopropeno (1-MCP)}

El 1-MCP $\left(\mathrm{C}_{4} \mathrm{H}_{6}\right)$ es una olefina cíclica, a temperatura y presión estándar, es un gas con un peso molecular de 54 (Blankenship y Dole, 2003). El 1-MCP ocupa los receptores del etileno de manera irreversible, bloqueando la cascada de transducción de señales que conllevan a la expresión de genes relacionados con la respuesta al etileno (In et al., 2013). La afinidad del 1-MCP por los receptores es diez veces mayor a la del etileno y actúa a más bajas concentraciones, también regula la biosíntesis de etileno a través de la inhibición del proceso autocatalítico (Blankenship y Dole, 2003). El compuesto 
no es tóxico, es inodoro, estable a temperatura ambiente, además, es de fácil aplicación y altamente eficaz para proteger a muchas especies agrícolas de la acción del etileno, incluyendo, frutos, vegetales, flores cortadas y plantas en maceta (Serek et al., 2006; Watkings, 2006; Huber, 2008). A pesar de que la unión del 1-MCP es irreversible, se ha reportado que los tejidos pueden recuperar la sensibilidad al etileno, debido a la capacidad de síntesis de nuevos receptores (Cameron y Reid, 2001), lo cual depende de la especie, tejido, estado de desarrollo y ambiente (Varanasi et al., 2013).

La concentración de 1-MCP necesaria para bloquear la acción del etileno varía de acuerdo con la especie, cultivar, estado de maduración, capacidad de producción de nuevos receptores, tiempo y temperatura de exposición (Watkins, 2006). Las dosis óptimas varían entre especies, pero Blankenship y Dole (2003) reportan diferentes concentraciones y temperaturas para la aplicación de 1-MCP, las cuales se encuentran entre 0,1 a $100 \mu \mathrm{L} \mathrm{L}^{-1}$ a $20-25^{\circ} \mathrm{C}$ por 6 a $24 \mathrm{~h}$. Sin embargo, la concentración recomendada para productos de uso comercial (EthylBloc ${ }^{\circledR}$ y ${ }^{\mathrm{T}}$ SmartFresh) está entre 100 a $500 \mu \mathrm{L} \mathrm{L}^{-1}$, alrededor de mil veces mayor, probablemente debido a una alta posibilidad de pérdidas del 1-MCP (Serek et al., 2006).

\section{Efecto de la aplicación de 1-MCP}

El 1-MCP ha mostrado un efecto significativo en la disminución de la maduración y senescencia de diversos productos agrícolas, como frutos, hortalizas y especies ornamentales (Serek et al., 2006; Watkins, 2006). Los mejores resultados se han observado en frutos climatéricos (Blankenship y Dole, 2003; Hubert, 2008) aunque, en frutos no climatéricos también se han encontrado resultados favorables (Dou et al., 2005).

El 1-MCP disminuye la producción de etileno (Ma et al., 2009; Cerqueira et al., 2009; Choi et al., 2008; Zhang et al., 2012), debido a que afecta su síntesis autocatalítica al disminuir la expresión de genes que codifican para las enzimas ACS y ACO. Por ejemplo, el 1-MCP disminuye la expresión de los genes ACS1, ACO1 y ACO2 en manzano (Yang et al., 2013), banano (Golding et al., 1999), tomate (Klee y Giovannoni, 2011), durazno (Zhang et al., 2012) y brócoli (Gang et al., 2009). A su vez, el 1-MCP también afecta la señalización del etileno, debido a que disminuye la expresión de genes que codifican para receptores de etileno, como BoERS; BoETR1; BoETR2 en brócoli (Gang et al., 2009); PaERS1 en aguacate (Owino et al., 2002), ETR1, ETR2, ETR5, ERSs, además de CTR1, EIN2A, EIL4 y ERFs en manzana (Yang et al., 2013).

El 1-MCP afecta la biosíntesis, señalización, y por tanto, los procesos que dependen de etileno durante la maduración. El 1-MCP disminuye la pérdida de firmeza en frutos de pera (Villalobos et al., 2011), ciruela (Khan y Singh, 2007), tomate (Choi et al., 2008), guayaba (Cequeira et al., 2009), banano (Lohani et al., 2004), kiwi (Boquete et al., 2004), aguacate (Jeong y Hubert, 2004), fresa (Balogh et al., 2005), entre otros. Lo anterior se debe a que el 1-MCP disminuye la actividad de enzimas que degradan pared celular, como pectin-metilesterasa (PME), poligalacturonasa (PG), endo- $\beta-1,4$-glucanasa (EGasa) y pectato liasa (PL) (Lohani et al., 2004; Khan y Singh, 2007), además de expansinas (EXP), $\beta$-galactosidasa, $\alpha$-arabinofuranosidasa y $\beta$-xilosidasa (Boquete et al., 2004). A su vez, la disminución de la actividad de dichas enzimas está relacionada con la disminución de la expresión de genes AcXET1 (xyloglucano endotransglicosilasas), AcEXP, AcEXP3 en chirimoya (Li et al., 2009), MaEXP1 en banano (Trivedi y Nath, 2004) y EGasa y plB (pectato liasa B) en fresa (Balogh et al., 2005).

Estudios recientes muestran que el 1-MCP disminuye la actividad de enzimas clorofilasas, manteniendo el color verde en el brócoli (Yuan et al., 2010) y col china (Sun et al., 2012). La dis- 
minución de la degradación de clorofilas puede atribuirse a la inhibición de la expresión de los genes PAO (feoforbida oxigenasa), NYC ("nonyellow colorrriiing"), NOL ("NYC1-like") y SGR1 ("stay green 1"), los cuales están estrechamente relacionados con la vía catabólica de las clorofilas (Cheng et al., 2012). Además, el 1-MCP disminuye la acumulación de licopeno en tomate (Zhang et al., 2009), y retrasa el desarrollo de color en frutos como pepino (Nilsson, 2005), pera (Villalobos et al., 2011) y tomate (Zhang et al., 2009).

También se ha reportado que el 1-MCP disminuye el contenido de azúcares y degradación de ácidos orgánicos en frutos de guayaba (Singh y Pal, 2008), tomate (Zhang et al., 2009), pitahaya amarilla (Deaquiz et al., 2014), granadilla (Dussan et al., 2011), entre otros. Con lo anterior, se puede afirmar que el 1-MCP afecta el metabolismo de carbohidratos y ácidos orgánicos, Mainardi et al. (2006) reportan que el 1-MCP disminuye la actividad de enzimas fosforilasas de almidón ( $\alpha$-1,4-Glucano-fosforilasa), la expresión de genes PPAIV1 (invertasa ácida soluble) y PpSPS1 (sacarosa fosfato sintasa), con lo cual, la pérdida de sacarosa y acumulación de hexosas en frutos de pera se reduce (Itai y Tanahashi, 2008).

El contenido total de volátiles disminuye con la aplicación de 1-MCP, pero los volátiles individuales son afectados diferencialmente (Kondo et al., 2005). En frutos de manzana, la producción de ésteres, alcoholes y aldehídos fue inhibida por el 1-MCP (Mattheis et al., 2005). En frutos de melón el 1-MCP suprime la expresión de cuatro genes de alcohol-acil-transferasa y disminuye la actividad de ésta enzima (El-Sharkawy et al., 2005), no obstante, la enzima alcohol deshidrogenasa $(\mathrm{ADH})$ no es afectada por el 1-MCP en manzana (Defilippi et al., 2005).

El efecto del 1-MCP sobre la calidad nutricional de los productos agrícolas no ha sido estudiado a profundidad. Se ha encontrado que el 1-MCP puede disminuir la pérdida de vitamina $C$ en frutos de jujube chino (Jiang et al., 2004), pero no en frutos de guayaba (Cerqueira et al., 2009). Además, el 1-MCP incrementa el contenido de antioxidantes solubles en agua, pero no altera los compuestos fenólicos en frutos de manzana (Defilippi et al., 2005). A su vez, el 1-MCP disminuye la concentración de antocianinas y compuestos fenólicos en col china (Sun et al., 2012) y brócoli (Yuan et al., 2010). Estos reportes indican que la respuesta del 1-MCP sobre la calidad nutricional de los productos agrícolas es muy variable, poniendo en evidencia que está influenciada por diversos factores (Watkins, 2006).

\section{Sales de plata}

El ion plata $\left(\mathrm{Ag}^{2+}\right)$ es un fuerte inhibidor de la acción del etileno (Taiz y Zeiger, 2006). El ion $\mathrm{Ag}^{2+}$ remplaza al $\mathrm{Cu}$ presente en los receptores de etileno (Serek et al., 2006), en consecuencia se afecta la señalización inducida por etileno y se disminuyen los procesos de maduración y senescencia (Knee, 1995). Los primeros estudios se realizaron en flores de corte con nitrato de plata $\left(\mathrm{AgNO}_{3}\right)$ (Kofranek y Paul, 1975), sin embargo, se encontró que este compuesto es tóxico en aplicaciones al follaje y su translocación en tallos es lenta (Staby et al., 1993). El tiosulfato de plata (STS; $\mathrm{Ag}\left(\mathrm{S}_{2} \mathrm{O}_{3}\right)_{2}{ }^{3-}$ ) es más eficiente, debido a que no es fitotóxico y su movilidad en los tejidos de la planta es mayor (Veen, 1983).

El STS se ha utilizado ampliamente en la poscosecha de flores de corte en la sabana de Bogotá (Colombia) y en varias especies de orquídeas, debido a su eficacia para disminuir la senescencia y prolongar la vida en florero (López et al., 2008). El tratamiento con STS se realiza por medio de soluciones de hidratación "tratamiento de carga" a dosis de $1 \mu \mathrm{M}$ de $\mathrm{Ag}^{2+}$ por tallo (Newman et al., 1998), utilizando compuestos a base de STS, como Chrysal AVB, Floríssima 125 y Florissant 100 (López et al., 2008). Es importante tener en cuenta que la eficiencia de los 
tratamientos a base de STS varía en función de la sensibilidad de las distintas especies al etileno exógeno y en un buen número de ellas, el tratamiento de carga no se justifica (Armitage y Laushman, 2003). La utilización de STS se ha restringido en algunos países para plantas en macetas, debido a los efectos tóxicos en el ambiente y contaminación de aguas subterráneas (Serek y Reid, 1993), además en productos alimenticios, el STS es totalmente prohibido (Chen et al., 2008).

\section{Aminoetoxi-vinil-glicina (AVG)}

El AVG es un compuesto análogo etoxi de rizobitoxina que inhibe la biosíntesis de etileno, debido a que bloquea la acción de la enzima ACC sintasa, mediante la unión al sustrato (fosfato de piridoxal: PLP) (Johnson y Colgan, 2003). La aplicación de AVG ha mostrado resultados favorables en la disminución de la caída de frutos y retraso de la maduración (Schupp et al., 2004) en frutales como manzano (Salas et al., 2011), durazno (Hayama et al., 2008), nectarines (McGlasson et al., 2005) y peral (D'Aquino et al., 2010), con efectos diferenciales dependiendo del cultivar (Belding y Lokaj, 2002).

La aplicación de AVG puede realizarse en precosecha (foliar y drench) y poscosecha (inmersión de los frutos). Las dosis para aplicaciones foliares se encuentran alrededor de $124 \mathrm{~g} \mathrm{ha}^{-1}$; 5-20 $\mu \mathrm{M}$ para aplicación en drench y $1 \mathrm{~g} \mathrm{~L}^{-1}$ para inmersión de tejidos (Saltveit, 2005). A pesar de su eficacia, este compuesto es ineficiente cuando los productos agrícolas tienen contacto con el etileno del ambiente (Newman et al., 1998).

Los tratamientos precosecha y poscosecha con AVG disminuyen la producción de etileno, la respiración, el ablandamiento, la degradación de clorofilas, la pérdida de peso, en general retrasan el proceso de maduración en frutos climatéricos (Hayama et al., 2008) y flores de corte (Seo et al., 2009). Algunos estudios muestran que el AVG no afecta negativamente las carac- terísticas organolépticas de los frutos, sin embargo, puede alterar del desarrollo de aroma, debido a la disminución del contenido de volátiles (Salas et al., 2011; Romani et al., 1983). Además, el AVG puede disminuir algunos desórdenes fisiológicos en los frutos, como el pardeamiento enzimático en pera (D'Aquino et al., 2010), no obstante, otros reportes mencionan que el AVG puede agravar otros desordenes fisiológicos en frutos de manzana, como "Core flush" y "Breakdown" (Johnson y Colgan, 2003).

\section{Ácido aminooxiacético (AOA)}

E1 AOA es un compuesto inhibidor no especifico de todas las enzimas que requieren fosfato de piridoxal como coenzima, incluyendo ACC sintasa, por lo cual, disminuye la síntesis de etileno endógeno (Taiz y Zeiger, 2006), pero no impide el efecto del etileno exógeno (López et al., 2008). Debido a que es un inhibidor no específico, puede interferir en numerosas reacciones enzimáticas, pudiendo afectar otros procesos fisiológicos (Staby et al., 1993). El AOA no es tóxico para el medio ambiente, por lo cual se considera como una alternativa viable ecológicamente en la poscosecha de productos agrícolas (Cubillos et al., 2001).

Los efectos de la aplicación de AOA en la poscosecha de flores son dependientes de la especie (López et al., 2008). Shinozaki et al. (2011) confirmaron la inhibición de la producción de etileno mediante tratamientos con $5 \mathrm{mM}$ de AOA en pétalos de Violeta. La inmersión de tallos de alstroemeria cultivar Diamond en una solución de AOA durante 3 h estimuló mayor apertura floral (Hernández et al., 2006), pero Shimamura et al. (1997) no encontraron efecto favorable en la disminución de la senescencia de Limonium, incluso el AOA causó pardeamiento de los tallos e inhibió la apertura de los floretes. El AOA puede generar resultados controversiales a bajas concentraciones, sin embargo se ha reportado que el $\mathrm{AOA}$ es mil veces menos efectivo que el AVG (Nissen, 1994). 
Otro factor que afecta la eficacia del AOA es el $\mathrm{pH}$ de la solución de tratamiento, el cual debe estar alrededor de 3 para evitar crecimiento microbiano, además, la combinación de AOA y glucosa tienen un efecto sinérgico, aumentando la vida en florero en Dendrobium (Rattanawisalanon et al., 2003). Resultados similares fueron reportados previamente en clavel (Yakimova et al., 1997). En Colombia, Cubillos et al. (2001) concluyeron que la aplicación del producto Florissima 135 (ingrediente activo $\mathrm{AOA}$ ), con un tiempo de exposición de $4 \mathrm{~h}$, es la mejor alternativa para remplazar los tratamientos a base de plata. Resultados similares fueron encontrados con $10 \mathrm{mg} \mathrm{L}^{-1}$ de SR-AOA en clavel (López et al., 2008).

\section{Permanganato de potasio $\left(\mathrm{KMnO}_{4}\right)$}

$\mathrm{E} 1 \mathrm{KMnO}_{4}$ es un agente oxidante fuerte en muchas reacciones redox, cuando éste compuesto entra en contacto con el etileno, lo oxida, produciendo $\mathrm{H}_{2} \mathrm{O}, \mathrm{CO}_{2}, \mathrm{MnO}_{2}$ y $\mathrm{KOH}$ (Wills y Warton, 2004), lo cual disminuye los niveles de etileno en la atmósfera y por tanto afecta los procesos dependientes de etileno durante la maduración (Sammi y Masud, 2007). Se han realizado estudios con resultados favorables en frutos climatéricos, como banano (Chauhan et al., 2006), mango y aguacate (Illeperuma y Jayasuriya, 2002), tomate (Salamanca et al., 2014), manzana (Brackmann et al., 2006), bananito (García et al., 2012), papaya (Silva et al., 2009) y en frutos no climatéricos, como fresa (Wills y Kim, 1995).

Para que el $\mathrm{KMnO}_{4}$ sea efectivo, debe ser adsorbido a un vehículo (carrier) para formar un absorbente sólido que aumente el área de contacto, debido a que las únicas fuerzas que actúan para que ocurra el contacto absorbente-etileno son la difusión y convección natural del aire en la atmósfera (Wills y Warton, 2004). Algunos de los carriers más utilizados son la zeolita, vermiculita, alúmina, perlita (Esguerra et al., 1978; Wills y Warton, 2004), entre otros. Los carriers de menor densidad y mayor área superficial presentan mayor capacidad para absorber el $\mathrm{KMnO}_{4}$ y en consecuencia, mayor eficiencia en la reducción de los niveles de etileno (Kavanagh y Wade, 1987).

Existen numerosos estudios publicados sobre el uso de $\mathrm{KMnO}_{4}$ en la poscosecha, no obstante, la información con respecto a la dosis y la cantidad de etileno que puede oxidar el $\mathrm{KMnO}_{4}$ no es clara (Wills y Warton, 2004). Los resultados en la poscosecha con el uso de $\mathrm{KMnO}_{4}$ dependen de la especie, carrier, empaque del producto $y$ de las condiciones de almacenamiento (humedad relativa y temperatura) (Bal y Celik, 2010).

El $\mathrm{KMnO}_{4}$ disminuye la tasa respiratoria, pérdida de peso y producción etileno en frutos de zapote (Bhutia et al., 2011), tomate (Sammi y Masud, 2007), papaya (Silva et al., 2009), sin embargo, muchas veces la tasa de emisión de etileno es superior a la tasa de oxidación (Amarante y Steffens, 2009a). Por otro lado, el $\mathrm{KMnO}_{4}$ disminuye el ablandamiento e incrementa la vida poscosecha en manzana (Amarante y Steffens, 2009a), kiwi (Bal y Celik, 2010), mango (Illeperuma y Jayasuriya, 2002) y papaya (Corrêa et al., 2005). También se ha encontrado que el $\mathrm{KMnO}_{4}$ en banano mantiene la acidez total titulable (ATT) y disminuye la tasa de incremento de los sólidos solubles totales (SST), debido a la disminución de la actividad de la enzima sacarosa fosfato sintasa (Choudhury et al., 2009). Además, el $\mathrm{KMnO}_{4}$ disminuye la relación de madurez (SST/ATT) en manzana (Amarante y Steffens, 2009b), bananito (García et al., 2012), entre otros.

\section{CONCLUSIONES}

A partir del conocimiento que se tiene en la fisiología del etileno en la poscosecha, se han desarrollado una serie de métodos para prevenir el deterioro de los productos agrícolas. Lo anterior se ha logrado mediante la inhibición 
de la biosíntesis, acción (percepción y señalización) y eliminación del etileno del ambiente. Los compuestos AVG y AOA inhiben la enzima ACC sintasa y disminuye la producción de etileno, sin embargo, no pueden prevenir el efecto del etileno presente en el ambiente. Las sales de plata afectan la acción del etileno por unirse a los receptores, no obstante, presentan serias limitaciones debido a su toxicidad para el ambiente. El 1-MCP también se une a los receptores, siendo uno de los inhibidores de la acción de etileno más estudiados y con mayor potencial en la poscosecha, debido a su alta eficacia en disminuir la maduración y senescencia.
El permanganato de potasio es un oxidante del etileno, con resultados favorables para mejorar la poscosecha de productos agrícolas.

\section{AGRADECIMIENTOS}

Los autores expresamos nuestros más sinceros agradecimientos al Ing. Agr., M.Sc. Fredy Alexánder Salamanca Gutiérrez (qepd), quién en vida contribuyó significativamente al manejo poscosecha de productos agrícolas en Colombia. Fredy, paz en tu tumba, permanecerás por siempre en nuestra memoria.

\section{REFERENCIAS BIBLIOGRÁFICAS}

Adams-Phillips, L., C. Barry y J. Giovannoni. 2004. Signal transduction systems regulating fruit ripening. Trends Plant Sci. 9(7), 331-338. Doi: 10.1016/j. tplants.2004.05.004

Amarante, C.V. y C. Steffens. 2009a. O tratamento précolheita com AVG, aliado à absorção do etileno durante oarmazenamento refrigerado, preserva a qualidade de maçãs 'Gala'. Rev. Bras. Frutic. 31(2), 334-342. Doi: 10.1590/S0100-29452009000200007

Amarante, C.V. y C.A. Steffens. 2009b. Sachês absorvedores de etileno na pós-colheita de maçãs 'Royal Gala'. Rev. Bras. Frutic. 31(1), 71-77. Doi: 10.1590/ S0100-29452009000100011

Armitage, A. y J. Laushman. 2003. Specialty cut flowers. Timber Press, Portland, OR.

Arora, A. 2008. Biochemistry of flower senescence. pp. 51-85. En: Paliyath, G., D. Murr., A. Handa y S. Lurie (eds.). Postharvest biology and technology of fruits, vegetables, and flowers. Wiley-Blackwell Publishing, New Delhi, India.

Bal, E. y S. Celik. 2010. The effects of postharvest treatments of salicylic acid and potassium permanganate on the storage of kiwifruit. Bulg. J. Agric. Sci. 16(5), 576-584.

Balogh, A., T. Koncz, V. Tisza, E. Kiss y L. Heszky. 2005. The effect of 1-MCP on the expression of several ripening-related genes in strawberries. HortScience 40(20), 88-90.
Bapat, V.A., P.K. Trivedi, A. Ghosh, V.A. Sane, T.R. Ganapathi y P. Nath. 2010. Ripening of fleshy fruit: Molecular insight and the role of ethylene. Biotechnol. Adv. 28, 94-107. Doi: 10.1016/j.biotechadv.2009.10.00

Barry, C. y J. Giovannoni. 2007. Ethylene and fruit ripening. J. Plant. Growth. Regul. 26, 143-159.

Belding, R. y G. Lokaj. 2002. Aminoethoxyvinylglycine treatment of peach fruit reduces ethylene production and softening. HortScience 37, 1065-1068.

Bhutia, W., R. Pal, S. Sen y S. Jha. 2011. Response of different maturity stages of sapota (Manilkara achras) cv. Kallipatti to in-package ethylene absorbent. J. Food Sci. Technol. 48(6), 763-768. Doi: 10.1016/j.postharvbio.2003.09.013

Binder, B. 2008. The ethylene receptors: complex perception for a simple gas. Plant Sci. 175, 8-17. Doi: 10.1016/j.plantsci.2007.12.001

Blankenship, S. y J. Dole. 2003. 1-Methylcyclopropene: a review. Postharv. Biol. Technol. 28, 1-25. Doi: 10.1016/S0925-5214(02)00246-6

Boquete, E., G. Trinchero, A. Fraschina, F. Vilella y G. Sozzli. 2004. Ripening of 'Hayward' kiwifruit treated with 1-methylcyclopropene after cold storage. Postharv. Biol Technol. 32, 57-65. Doi: 10.1016/j.postharvbio.2003.09.013 
Bouzayen, M., A. Latché. P. Nath y J. Pech. 2010. Mechanism of fruit ripening. pp. 319-339. En: Pua, E. y M. Davey (eds.). Plant developmental biology-biotechnological perspectives. Springer-Verlag, Berlín.

Brackmann, A., R. Giehl, S. Freitas, A. Eisermann y A. Mello. 2006. Uso de filmes de polietileno e absorção de etileno para o transporte refrigerado de maçã 'Gala'. Semin. Ciênc. Agrár. 27(3), 423-428.

Cameron, A. y M. Reid. 2001. 1-MCP blocks ethyleneinduced petal abscission of Pelargonium peltatum but the effect is transient. Postharv. Biol. Technol. 22, 169-177. Doi: 10.1016/S0925-5214(00)00189-7

Cerqueira, T., A. Jacomino, F. Sasaki y L. Amorim. 2009. Controle do amadurecimento de goiabas 'Kumagai' tratadas com 1-metilciclopropeno. Rev. Bras. Frutic. 31(3), 687-692. Doi: 10.1590/S01002945200900030001

Chauhan, O., P. Raju, D. Dasgupta y A. Bawa. 2006. Modified atmosphere packaging of banana (cv. Pachbale) with ethylene, carbon dioxide and moisture scrubbers and effect on its ripening behaviour. Amer. J. Food Technol. 1(2), 179-189.

Chen, Y., L. Chen y J. Shaw. 2008. Senescence-associated genes in harvest broccoli florets. Plant Sci. 175, 137-144. Doi: 10.1016/j.plantsci.2008.03.007

Chen, Y., N. Etheridge y E. Schaler. 2005. Ethylene signal transduction. Ann. Bot. 95, 901-915. Doi: 10.1093/aob/mci100

Cheng, Y., Y. Dong, H. Yan, W. Ge, C. Shen, J. Guan, L. Liu y Y. Zhang. 2012. Effects of 1-MCP on chlorophyll degradation pathway-associated genes expression and chloroplast ultrastructure during the peel yellowing of Chinese pear fruits in storage. Food Chem. 135(2), 415-422. Doi: 10.1016/j.foodchem.2012.05.017

Choi, S., P. Tsouvaltzis, C. Lim y D.J. Hubert. 2008. Suppression of ripening and induction of asynchronous ripening in tomato and avocado fruits subjected to complete or partial exposure to aqueous solutions of 1-methylcyclopropene. Postharv. Biol. Technol. 48, 206-214. Doi: 10.1016/j.postharvbio.2007.10.008

Choudhury, S., S. Roy y D. Sengupta. 2009. A comparative study of cultivar differences in sucrose phosphate synthase gene expression and sucrose formation during banana fruit ripening. Postharv. Biol. Technol. 54, 15-24. Doi: 10.1016/j.postharvbio.2009.05.003

Corrêa, S., M. Filho, M. Silva, J. Oliveira, E. Aroucha,R. Silva, M. Pereira y H. Vargas. 2005. Effect of the potassium permanganate during papaya fruit ripe- ning: Ethylene production. J. Phys. IV France 125 869-871. Doi: 10.1051/jp4:2005125201

Cubillos, E., V. Molina, V.J. Flórez y G. Fischer. 2001. Efecto inhibidor de etileno en la longevidad floral del clavel (Dianthus caryophyllus) como probables sustitutos del tiosulfato de plata (STS). Agron. Colomb. 18, 7-13.

Czarny, J., V. Grichko y B. Glick. 2006. Genetic modulation of ethylene biosynthesis and signaling in plants. Biotechnol. Adv. 24, 410-419. Doi 10.1016/j.biotechadv.2006.01.003

D’Aquino, S., M. Schirra, M. Molinu, M. Tedde y A. Palma. 2010. Preharvest aminoethoxyvinylglycine treatments reduce internal browning and prolong the shelf-life of early ripening pears. Sci. Hortic. 125, 353-360. Doi: 10.1016/j.scienta.2010.04.020

Deaquiz, Y., J. Álvarez-Herrera y G. Fischer. 2014. Ethylene and 1-MCP affect the postharvest behavior of yellow pitahaya fruits (Selenicereus megalanthus Haw.). Agron. Colomb. 32(1), 44-51.

Defilippi, B., A. Kader y A. Dandekar. 2005. Apple aroma: alcohol acyltransferase, a rate limiting step for ester biosynthesis, is regulated by ethylene. Plant Sci. 168, 199-210. Doi: 10.1016/j.plantsci.2004.12.018

Dou, H., S. Jones y M. Ritenour. 2005. Influence of 1-MCP application and concentration on post-harvest peel disorders and incidence of decay in citrus fruit. J. Hort. Sci. 80(7), 86-92.

Dussan, S., L. Serna y A. Perenguez. 2011. Efecto de la aplicación de 1-Metilciclopropeno sobre algunas propiedades físico-químicas y organolépticas del fruto de la granadilla. Acta Agron. 60(3), 237-244.

El-Sharkawy, I., D. Manriquez, F. Flores, F. Regad, M. Bouzayen y A. Latche. 2005. Functional characterization of a melon alcohol acyl-transferase gene family involved in the biosynthesis of ester volatiles. Identification of the crucial role of a threonine residue for enzyme activity. Plant. Mol. Biol. 59, 345-62. Doi: 10.1007/s11103-005-8884-y

Esguerra, E., D. Mendoza y E. Pantastico. 1978. Regulation of fruit ripening. II. Use of perlite- $\mathrm{KMnO}_{4}$ as an ethylene absorbent. Phillip. J. Sci. 107, 23-31.

Gang, M., W. Ran., W. Cheng-Rong, K. Masaya, Y. Kazuki, Q. Fei-fei y X. Hui-Lian. 2009. Effect of 1-methylcyclopropene on expression of genes for ethylene biosynthesis enzymes and ethylene receptors in post-harvest broccoli. Plant Growth Regul. 57, 223-232. Doi: 10.1007/s10725-008-9339-7

García, J., H. Balaguera-López y A. Herrera. 2012. Conservación del fruto de banano bocadillo (Musa AA 
Simmonds) con la aplicación de permanganato de potasio $\left(\mathrm{KMnO}_{4}\right)$. Rev. Colomb. Cienc. Hortic. 6(2), 161-171.

Golding, J., D. Shearer. S. Wyllie y W. McGlasson. 1999. Application of 1-MCP and propylene to identify ethylene-dependent ripening processes in mature banana fruit. Postharv. Biol. Technol. 14, 87-98. Doi: 10.1016/S0925-5214(98)00032-5

Hayama, H., M. Tatsuki y Y. Nakamura. 2008. Combined treatment of aminoethoxyvinylglycine (AVG) and 1-methylcyclopropene (1-MCP) reduces melting-flesh peach fruit softening. Postharv. Biol. Technol. 50, 228-230. Doi: 10.1016/j.postharvbio.2008.05.003

Hernández, A.D., J.M. Pinedo, M.T. Colinas, J. Meza y S. Juárez. 2006. Conservación de flores de alstroemeria (Alstroemeria spp.) mediante soluciones preservativas en poscosecha. Rev. Chapingo Ser. Hortic. 12(1), 19-25.

Hubert, D. 2008. Suppression of ethylene responses through application of 1-methylcyclopropene: a powerful tool for elucidating ripening and senescence mechanisms in climacteric and nonclimacteric fruits and vegetables. HortScience 43(1), 106-111.

Illeperuma, C. y P. Jayasuriya. 2002. Prolonged storage of 'Karuthacolomban' mango by modified atmosphere packaging at low temperature. J. Hort. Sci. Biotechnol. 77(2), 153-157. Doi: 10.4038/jnsfsr. v32i1-2.2424

In, B.C., J. Strablea, B.M. Binder, T.G. Falbel y S.E. Patterson. 2013. Morphological and molecular characterization of ethylene binding inhibition in carnations. Postharv. Biol. Technol. 86, 272-279. Doi: 10.1016/j.postharvbio.2013.07.007

Itai, A. y T. Tanahashi. 2008. Inhibition of sucrose loss during cold storage in Japanese pear (Pyrus pyrifolia Nakai) by 1-MCP. Postharv. Biol. Technol. 48, 355363. Doi: 10.1016/j.postharvbio.2007.10.015

Jeong, J. y D. Hubert. 2004. Suppression of avocado (Persea americana Mill.) fruit softening and changes in cell wall matrix polysaccharides and enzyme activities: differential responses to 1-MCP and delayed ethylene application. J. Amer. Soc. Hort. Sci. 129, 752-759.

Jiang, Y. y J. Fu. 2000. Ethylene regulation of fruit ripening: Molecular aspects. Plant. Growth Regul. 30, 193-200. Doi: 10.1023/A:1006348627110

Jiang, W.B., Q. Sheng, Y.M. Jiang y X.J. Zhou. 2004. Effects of 1-methylcyclopropene and gibberellic acid on ripening of Chinese jujube (Zizyphus jujuba M.) in relation to quality. J. Sci. Food Agric. 84,3135. Doi: $10.1002 /$ jsfa.1594
Johnson, D. y R. Colgan. 2003. Low ethylene controlled atmosphere induces adverse effects on the quality of 'Cox's Orange Pippin' apples treated with aminoethoxyvinylglycine during fruit development. Postharv. Biol. Technol. 27, 59-68. Doi: 10.1016/ S0925-5214(02)00188-6

Kavanagh, E. y N. Wade. 1987. Role of the carrier in the removal of ethylene by permanganate from storage atmospheres. ASEAN Food J. 3, 128-134.

Kesari, R., P. Trivedi y P. Nath. 2007. Ethylene-induced ripening in banana evokes expression of defense and stress related genes in fruit tissue. Postharv. Biol. Technol. 46, 136-143. Doi: 10.1016/j.postharvbio.2007.04.010

Khan, A. y Z. Singh. 2007. 1-MCP regulates ethylene biosynthesis and fruit softening during ripening of 'Tegan Blue' plum. Postharv. Biol. Technol. 43, 298-306. Doi: 10.1016/j.postharvbio.2006.10.005

Klee, H. y J. Giovannoni. 2011. Genetics and control of tomato fruit ripening and quality attributes. Annu. Rev. Genet. 45, 41-59. Doi: 10.1146/annurev-genet-110410-132507

Knee, M. 1995. Copper reverses silver inhibition of flower senescence in Petunia hybrida. Postharv. Biol. Technol. 6, 121-128. Doi: 10.1016/09255214(94)00038-T

Kofranek, A. y J. Paul. 1975. The value of impregnating cut stems with high concentrations of silver nitrate. Acta Hort. 41,199-206.

Kondo, S., S. Setha, D. Rudell, D. Buchanan y J. Mattheis. 2005. Aroma volatile biosynthesis in apples affected by 1-MCP and methyl jasmonate. Postharv. Biol. Technol. 36, 61-68. Doi: 10.1016/j. postharvbio.2004.11.005

Li, C., W. Shen, W. Lu. Y. Jiang. J. Xie y J. Chen. 2009. 1-MCP delayed softening and affected expression of XET and EXP genes in harvested cherimoya fruit. Postharv. Biol. Technol. 52, 254-259. Doi: 10.1016/j.postharvbio.2008.12.009

Lohani, S., P. Trivedi y P. Nath. 2004. Changes in activities of cell wall hydrolases during ethyleneinduced ripening in banana: effect of 1-MCP, ABA and IAA. Postharv. Biol. Technol. 31, 119-26. Doi: 10.1016/j.postharvbio.2003.08.001

López, P., D. Neisa, C. Bacca y V. Flórez. 2008. Evaluación de preservantes florales en la poscosecha de tres variedades de clavel estándar. Agron. Colomb. 26(1), 116-126.

Ma, G., R. Wang, C. Wang, M. Kato, K. Yamawaki, F. Oin y H. Xu. 2009. Effect of 1-methylcyclopropene on expression of genes for ethylene biosynthesis 
enzymes and ethylene receptors in post-harvest broccoli. Plant Growth. Regul. 57, 223-232. Doi: 10.1007/s10725-008-9339-7

Mainardi, J., E. Purgatto, A. Jr. Vieira, W.A. Bastos, B.R. Cordenunsi, J.R. Oliveira y F.M. Lajolo. 2006. Effects of ethylene and 1-methylcyclopropene (1MCP) on gene expression and activity profile of $\alpha-1,4$-glucan-phosphorylase during banana ripening. J. Agric. Food Chem. 54(19), 7294-7299. Doi: 10.1021/jf061180k

Mattheis, J., X. Fan y L. Argenta. 2005. Interactive responses of Gala apple fruit volatile production to controlled atmosphere storage and chemical inhibition of ethylene action. J. Agric. Food. Chem. 53, 1-6. Doi: 10.1021/jf050121o

McGlasson, W., A. Rath y L. Legendre. 2005. Preharvest application of aminoethoxyvinylglycine (AVG) modifies harvest maturity and cool storage life of 'Arctic Snow' nectarines. Postharv. Biol. Technol. 36, 93-102. Doi: 10.1016/j.postharvbio.2004.10.006

Newman. J., L. Dodge y M. Reid. 1998. Evaluation of ethylene inhibitors for postharvest treatment of Gypsophila paniculata. HortTechnol. 8(1), 58-63.

Nilsson, T. 2005. Effects of ethylene and 1-MCP on ripening and senescence of European seedless cucumbers. Postharv. Biol. Technol. 36, 113-125. Doi: 10.1016/j.postharvbio.2004.11.008

Nissen, P. 1994. Stimulation of somatic embryogenesis in carrot by ethylene biosynthesis and action. Physiol. Plant. 92, 397-403. Doi: 10.1111/j.1399. 3054.1994.tb08827.x

Owino, W., R. Nakano, Y. Kubo y A. Inaba. 2002. Diferencial regulation of genes encoding ethylene biosynthesis enzymes and ethylene response sensor ortholog during ripening and response to wounding avocados. J. Amer. Soc. Hort. Sci. 127(4), 520-527.

Rattanawisalanon, C., S. Ketsa y W. Van Doorn. 2003. Effect of aminooxyacetic acid and sugars on the vase life of Dendrobium flowers. Postharv. Biol. Technol. 29, 93-100. Doi: 10.1016/S09255214(02)00242-9

Romani, R., J. Labavith, T. Yamashita, B. Hess y H. Rae. 1983. Preharvest AVG treatment of 'Bartlett' pear fruits: effects on ripening, color change, and volatiles. J. Amer. Soc. Hort. Sci. 108, 1046-1049.

Salamanca, F., H.E. Balaguera y A.O. Herrera. 2014. Efecto del permanganato de potasio sobre algunas características poscosecha de frutos de tomate "chonto" (Solanum lycopersicum L.). Acta Hort. 1016, 171-176.
Salas, N., F. Molina-Corral, G. González-Aguilar, A. Otero, D. Sepulveda y G. Olivas. 2011. Volatile production by 'Golden Delicious' apples is affected by preharvest application of aminoethoxyvinylglycine. Sci. Hortic. 130, 436-444. Doi: 10.1016/j. scienta.2011.07.017

Saltveit, M. 2005. Aminoethoxyvinylglycine (AVG) reduces ethylene and protein biosynthesis in excised discs of mature-green tomato pericarp tissue. Postharv. Biol. Technol. 35, 183-190Doi: 10.1016/j. postharvbio.2004.07.002

Sammi, S. y T. Masud. 2007. Effect of different packaging systems on storage life and quality of tomato (Lycopersicon esculentum var. Rio Grande) during different ripening stages. J. Food Safety 9, 37-44.

Sang-Dong, Y., C. Younghee y S. Jen. 2009. Emerging connections in the ethylene signaling network. Trends Plant Sci. 14(5), 270-279. Doi: 10.1016/j. tplants.2009.02.007

Schupp, J.R. y D.W. Greene. 2004. Effect of aminoethoxyvinylglycine (AVG) on preharvest drop, fruit quality, and maturation of 'McIntosh' apples. I. Concentration and timing of dilute applications of AVG. HortScience 5, 1030-1035.

Seo, S., S. Kang, I. Shim, W. Kim y S. Fujihara. 2009. Effects of various chemical agents and early ethylene production on floral senescence of Hibiscus syriacus. Plant Growth Regul. 57, 251-258. Doi: 10.1007/s10725-008-9342-z

Serek, M. y M. Reid. 1993. Anti-ethylene treatments for potted flowering plants-relative efficacy of inhibitors of ethylene action and biosynthesis. HortScience 28, 1180-1181.

Serek, M., E.J. Woltering, E.C. Sisler, S. Frello y S. Sriskandarajah. 2006. Controlling ethylene responses in flowers at the receptor level. Biotechnol. Adv. 24 368-381. Doi: 10.1016/j.biotechadv.2006.01.007

Shimamura, M., A. Ito, K. Suto, H. Okabayashi y K. Ichimura. 1997. Effects of $\alpha$-aminoisobutyric acid and sucrose on the base life of hybrid Limonium. Postharv. Biol. Technol. 12, 247-253. Doi: 10.1016/ S0925-5214(97)00062-8

Shinozaki, Y., T. Tanabata, I. Ogiwara, T. Yamada y M. Kanekatsu. 2011. Application of digital image analysis system for fine evaluation of varietal differences and the role of ethylene in visible petal senescence of morning glory. J. Plant Growth Regul. 30, 229-234. Doi: 10.1007/s00344-010-9182-8

Silva, D., L.C.C. Salomão, D.L. Siqueira, P.R. Cecon y A. Rocha. 2009. Potassium permanganate effects in postharvest conservation of the papaya cultivar 
Sunrise Golden. Pesq. Agropec. Bras. 44(7), 669-675. Doi: 10.1590/S0100-204X2009000700003

Singh, S. y R. Pal. 2008. Response of climacteric-type guava (Psidium guajava L.) to postharvest treatment with 1-MCP. Postharv. Biol. Technol. 47(3), 307314. Doi: $10.1016 /$ j.postharvbio.2007.08.010

Sisler, E. y M. Serek. 1997 Inhibitors of ethylene responses in plants at the receptor level: Recent developments. Physiol. Plant. 100, 577-582. Doi: 10.1111/j.1399-3054.1997.tb03063.x

Staby, G., R. Basel, M. Reld y L. Dodge. 1993. Efficacy of commercial anti-ethylene products for fresh cut flowers. HortTechnol. 3, 199-202.

Stepanova, A. y J. Alonso. 2009. Ethylene signaling and response: where different regulatory modules meet. Curr. Opin. Plant. Biol. 12, 548-555. Doi: 10.1016/j.pbi.2009.07.009

Sun, B., H. Yan, N. Liu, J. Wei y O. Wang. 2012. Effect of 1-MCP treatment on postharvest quality characters, antioxidants and glucosinolates of chinese kale. Food Chem. 131(2), 519-526.

Taiz, L. y E. Zeiger. 2006. Plant physiology. 4ª ed. Sinauer Associates, Sunderland, MA.

Trivedi, P. y P. Nath. 2004. MaExp1, an ethylene-induced expansin from ripening banana fruit. Plant Sci. 167, 1-8. Doi: 10.1016/j.plantsci.2004.07.005

Varanasi, V., S. Shin. F. Johnson. J. Mattheis y Y. Zhu. 2013. Differential suppression of ethylene biosynthesis and receptor genes in 'Golden Delicious' apple by preharvest and postharvest 1-MCP treatments. J. Plant. Growth Regul. 32, 585-595. Doi: 10.1007/s00344-013-9326-8

Veen, H. 1983. Silver thiosulphate: an experimental tool in plant science. Sci. Hortic. 20, 211-24. Doi: 10.1016/0304-4238(83)90001-8

Villalobos, M., W. Biasi, E. Mitcham y D. Holcroft. 2011. Fruit temperature and ethylene modulate 1-MCP response in Bartlee pears. Postharv. Biol. Technol. 60,17-23. Doi: 10.1016/j.postharvbio.2010.11.005

Watkins, C. 2006. The use of 1-methylcyclopropene (1MCP) on fruits and vegetables. Biotechnol. Adv. 24, 389-409. Doi: 10.1016/j.biotechadv.2006.01.005

Wills, R. y G. Kim. 1995. Effect of ethylene on postharvest life of strawberries. Postharv. Biol. Technol. 6 , 249-255. Doi: 10.1016/0925-5214(95)00005-O

Wills, R. y M. Warton. 2004. Efficacy of potassium permanganate impregnated into alumina beads to reduce atmospheric ethylene. J. Amer. Soc. Hort. Sci. 129(3), 433-438

Yakimova, E., B. Atanassova y V. Kapchina-Toteva. 1997. Longevity and some metabolic events in post-harvest spray carnation flowers. Bulg. J. Plant Physiol. 23, 57-65.

Yang, X., J. Song, L. Campbell-Palmer, S. Fillmore y Z. Zhang. 2013. Effect of ethylene and 1-MCP on expression of genes involved in ethylene biosynthesis and perception during ripening of apple fruit. Postharv. Biol. Technol. 78, 55-66. Doi: 10.1016/j. postharvbio.2012.11.012

Yuan, G., B. Sun, J. Yuan y M. Wang. 2010. Effect of 1-methylcyclopropene on shelf life, visual quality, antioxidant enzymes and health-promoting compounds in broccoli florets. Food Chem. 118, 774-781. Doi: 10.1016/j.foodchem.2009.05.062

Zhang, L., L. Jiang, Y. Shi, H. Luo, R. Kang y Z. Yu. 2012. Post-harvest 1-methylcyclopropene and ethephon treatments differently modify protein profiles of peach fruit during ripening. Food Res. Int. 48, 609-619. Doi: 10.1016/j.foodres.2012.05.022

Zhang, Z., D.J. Huber, B.M. Hurr y J. Rao. 2009. Delay of tomato fruit ripening in response to 1-methylcyclopropene is influenced by internal ethylene levels. Postharv. Biol. Technol. 54, 1-8. Doi: 10.1016/j.postharvbio.2009.06.003 